care that was responsible for the poor uptake. It was not due to massive apathy or recalcitrance, which we must confess, probably with other GPs, had been our mistaken impression in earlier years. Of the other two methods, one was the postal provision of data about their preventive care (much appreciated by the families incidentally), and only the other, the visiting of families in their homes, could be interpreted as "pressure." But all such visits had been carefully prearranged after much counselling by sensitive health visitors using non-judgmental negotiating skills and always respecting the right of the patient to decline. That some did is evidenced by our total lack of $100 \%$ scores.

Finally, the authors accuse us of making the less compliant families unpopular because they upset the statistics. Nothing can be further from the truth. The small number of non-compliers-a few of whom are persistently "apathetic" and "recalcitrant" despite all our efforts (as indeed are some of their endowed controls) - are accepted by us as they are: we will not stop counselling them we will hope to become more adept at it, and ultimately more successful, but in the mean time they will be no less popular; if anything the very reverse in that they provide a continuing challenge to us.

Dr Neil Colman (20 February, p 574) was worried about confidentiality with regard to contraception in our preventive care letters to households. We gave this much thought, and in fact did not mention contraception in the letters but almost always raised this topic when a patient came for other preventive care measures. We were also extremely cautious and carefully scrutinised records before mentioning cervical cytology in teenagers.

We hope these comments relieve the anxieties of our critics.

G N MARSH

Norton Medical Centre,

Stockton on Tees,

Cleveland TS20 lAN

\section{Tardive dyskinesia}

SIR,-In my article on tardive dyskinesia (16 January, p 150) I did not address the complex implications of the existence of similar abnorma movements in untreated psychotic patients. ${ }^{12} \mathrm{Dr}$ Trevor Turner raises this issue ( 5 March, p 719), apparently rehearsing the argument that tardive dyskinesia is not drug related but a feature of psychotic illness.

He offers descriptions of abnormal movements in psychiatric patients before the advent of antipsychotic drugs as evidence of tardive dyskinesia, but such historical reports require careful interpretation. Mackay ${ }^{3}$ and Marsden ${ }^{2}$ considered that the movements generally described were different in character from those of tardive dyskinesia. Furthermore, the terminology is confused, reflecting various notions of the cause of the motor phenomena observed. ${ }^{24}$ In addition, the patient population almost certainly included patients with organic disease such as encephalitis lethargica and syphilis, which can present with both psychotic features and motor disorder. ${ }^{25}$

An element of the historical perspective $\mathrm{Dr}$ Turner may have neglected is the number of reports that appeared soon after the introduction of antipsychotic drugs clearly describing tardive dyskinesia as a novel syndrome. ${ }^{6}$ The following decades have seen an increase in the prevalence of the condition accompanying the widespread use of these drugs, ${ }^{7}$ a finding not easily dismissed as simply a manifestation of increasing awareness of tardive dyskinesia.
Neither of the two studies cited by Dr Turner refers to the abnormal movements identified as tardive dyskinesia. One of the investigations compared inpatients with chronic schizophrenia with and without a history of antipsychotic drug treatment and found a similar prevalence of $a b$ normal involuntary movements. ${ }^{8}$ While this finding confirms the occurrence of spontaneous movement disorder in schizophrenia, the contribution of drug treatment is acknowledged in that "exposure to neuroleptics produced a striking increase in both the prevalence and severity of abnormal movements." Grouping movements into clinically recognisable syndromes showed a particular susceptibility to orofacial dyskinesia in the drug treated patients.

My article referred to advancing age and particular features of the schizophrenic illness as risk factors for the emergence of tardive dyskinesia. Antipsychotic drug treatment may interact with the disease process and age related cerebral deterioration to hasten or provoke the appearance of abnormal movements. ${ }^{10}$ Similarly, Dr Turner states that tardive dyskinesia results "primarily from the intrinsic disease process itself, mediated by dopamine antagonists." The specificity of such theories for schizophrenia, however, is challenged by the emerging evidence of increased susceptibility to early onset tardive dyskinesia in patients with affective disorder receiving antipsychotic drugs and the occurrence of tardive dyskinesia in non-psychotic patients treated with these drugs. ${ }^{10}$

In addition to tardive dyskinesia, schizophrenic patients taking antipsychotic medication may show other drug related movement disorders such as dystonia and akathisia. They can also exhibi stereotypies and mannerisms; a host of other abnormalities of posture, tone, and motor activity ${ }^{4}$ and possibly more subtle disturbances of motor function, ${ }^{11}$ all thought to be related to the illness The distinction between these types of movements and, more fundamentally, the dissociation of the effects of drug treatment from any specific contribution of the schizophrenic illness, is relevant to the important research and legal issue mentioned by Dr Turner. Progress in this subject, however, is unlikely to be advanced by adopting his rather indiscriminate application of the term tardive dyskinesia. The term should be confined to orofacial dyskinesia and choreiform trunk and limb dyskinesia where this has developed in association with the administration of dopamine antagonist drugs.

Department of Psychiatry,

Charing Cross and Westminster Medical School,

London W6 8RP

THOMAS R E BARNES

1 Barnes TRE, Liddle PF. Tardive dyskinesia: implications for schizophrenia? In: Schiff AA, Roth Sir Martin, Freeman HL, eds. Schizophrenia: new pharmacological and clinical developments. London: Royal Society of Medicine Services, 1985: ments. London: Royal Society of Medicine Services, 1985 . 81-7. (International Congress and Symposium Series, No 94 .)
Marsden CD. Is tardive dyskinesia a unique disorder? In: Casey DE, Chase TN, Christensen AV, Gerlach J, eds. Dyskinesiaresearch and treatment. Berlin: Springer Verlag, 1985:64-71.

3 Mackay AVP. Controversies in tardive dyskinesia. In: Marsde CD, Fahn S, eds. Neurology. Vol 2. Movement disorders. London: Butterworths, 1981:249-76.

Rogers D. The motor disorders of severe psychiatric illness: a conflict of paradigms. Br f Psychiatry 1985;147:221-32.

5 Farran-Ridge C. Some symptoms referable to the basal ganglia occurring in dementia praecox and epidemic encephalitis. fournal of Mental Science 1926;72:513-23.

6 Crane GE. Persistent dyskinesia. Br 7 Psychiatry 1973;122: 395-405.

7 Jeste DV, Wyatt RJ. Changing epidemiology of tardive dyskinesia: an overview. Am J Psychiatry 1981;138:297-309.

8 Owens DGC, Johnstone EC, Frith CD. Spontaneous involuntary disorders of movement: their prevalence, severity and distribution in chronic schizophrenics with and without treatment with neuroleptics. Arch Gen Psychiatry 1982;39: 452-61.

9 Owens DGC. Involuntary disorders of movement in chronic schizophrenia-the role of the illness and its treatment. In: Casey DE, Chase TN, Christensen AV, Gerlach J, eds.
1985:79-87.

10 Barnes TRE. The present status of tardive dyskinesia and akathisia in the treatment of schizophrenia. Psychiatr Dev 1987;4:301-19.

11 Manschrek TC, Maher BA, Rucklos ME, Vereen DR Disturbed voluntary motor activity in schizophrenic disorder. Psychol Med 1982;12:73-84

SIR,-In commenting on the leading article by Dr Thomas Barnes (16 January, p 150) Dr Trevor Turner (5 March, p 719) criticises its statement that tardive dyskinesia is a disorder of movement caused by neuroleptic drugs. He buttresses his dissent by referring to historical and more recent studies indicating involuntary movements in psychotic patients never exposed to such treatment. Curiously, both authors cite my own work in support of their contradictory analyses. ${ }^{1}$ Neither appears to have appreciated its fundamental point.

Research in tardive dyskinesia is one of the few areas of scientific inquiry where experimental studies are entertained when they fail to include what is usually considered to be an essential, but here virtually unobtainable, control group-that is, in this case, one of indistinguishable patients in whom psychotic illness has run its course in the absence of treatment with the presumed offending neuroleptic. The available evidence indicates that the baseline level of involuntary movement disorder in untreated psychotic illness has been underestimated, particularly in patients with severe chronic disease. Evidence also indicates, however, that this unappreciated baseline can be further raised by long term exposure to neuroleptic drugs. The likelihood of involuntary movements appears to increase with the extent of cognitive impairment, both in treated and in untreated populations, suggesting some common association with abnormalities in cerebral structure. ${ }^{12}$

Our view is that neuroleptics may not be the "cause" of tardive dyskinesia. Rather, they seem to hasten and exacerbate the appearance of in voluntary movements among patients who would probably manifest such movements spontaneously.

JOHN L WADDINGTON

Department of Clinical Pharmacology,

Royal College of Surgeons in Ireland

Dublin 2, Eire

Waddington JL. Tardive dyskinesia in schizophrenia and other disorders: associations with ageing, cognitive dysfunction and structural brain pathology in relation to neuroleptic exposure. Human Psychopharmacology 1987;2:11-22.

2 Waddington JL, Youssef HA, Dolphin C, Kinsella A. Cognitive dysfunction, negative symptoms and tardive dyskinesia in schizophrenis their association in relation to topography of schizophrenia: their association in relation to topography of Arch Gen Psychiatry 1987;44:907-12.

\section{Microvascular fragility at high altitude}

SIR,-D During a recent expedition to the Himalayas we investigated microvascular fragility by the technique of mucosal petechiometry, as used by Hunter $e t a l^{1}$ and Dr J S Milledge and others (27 February, p 610). A $1 \mathrm{~cm}$ suction cup was applied at a negative pressure of $200 \mathrm{~mm} \mathrm{Hg}$ to the buccal mucosa of the lower lip for one minute, and the petechiae produced were counted. In 23 subjects (21 men, two women, age 23-60) there was a significant increase in petechiae after a five day ascent from $3800 \mathrm{~m}$ to $4900 \mathrm{~m}$ (mean $1.8 v 6.5$, $\mathrm{p}<0.02 \mathrm{Wilcoxon}$ 's signed rank test). The number of petechiae increased in 14 subjects, decreased in five, and was unchanged in four.

There was no association between the number of petechiae and symptoms of altitude sickness or arterial oxygen tension. One person in whom the number of petechiae increased from one to $20 \mathrm{had}$ a single retinal haemorrhage; there were no other retinal haemorrhages. The presence of nail 
haemorrhages at high altitude ${ }^{2}$ was not related to microvascular fragility; at $4900 \mathrm{~m}$ splinter haemorrhages were seen in four climbers with less than 15 petechiae and in none of four with more than 20 petechiae. Nail and retinal haemorrhages were not confined to those suffering from acute mountain sickness

In our study microvascular fragility did appea to be a feature of high altitude exposure, supporting the view of Hunter et al. ${ }^{1}$ The slow ascent and greater altitude attained by our expedition mem bers may account in part for the difference between our results and those of Dr Milledge and others. In eight of our climbers tested at sea leve the mean petechiae count was one and no increase in petechiae was observed after ascent to $3800 \mathrm{~m}$. In the authors' group a mean petechiae count of 11.5 was seen at $1300 \mathrm{~m}$ altitude; no sea level data were presented.

In the absence of an association with altitude sickness symptoms or arterial oxygen tension it is unlikely that increased microvascular fragility is implicated in the pathogenesis of acute mountain sickness. Microvascular fragility may be a characteristic of certain people at high altitude rather than characteristic of high altitude sickness.

P J G Forster

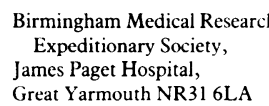

1 Hunter DJ, Smart JR, Whitton L. Increased capillary fragility a high altitude. Br Med f 1986;292:98.

2 Heath D, Williams DR. Man at high altitude. Edinburgh Churchill Livingstone, 1977.

\section{Crohn's disease presenting as anorexia nervosa}

SIR,-The title of the paper by Drs A P Jenkins and others (5 March, p 699) is misleading, because the patients they describe did not suffer from anorexia nervosa and did not present with symptoms of anorexia nervosa. They point out that the American Psychiatric Association's Diagnostic and Statistical Manual includes five criteria for the diagnosis of anorexia nervosa but did not add that all of these are required. The four cases reported satisfied only one of these criteria-weight loss, which is a symptom of very low diagnostic specificity. It can occur in a wide range of physica disorders which may present in young women, and in addition to Crohn's disease these include for example, malignant disease, diabetes, and pulmonary tuberculosis. It is also a common symptom in other mental illnesses, particularly depressive disorders.

The other criteria for diagnosis, particularly disturbance of body image and refusal to maintain body weight, which involves extreme voluntary measures to lose weight, have much more diag nostic specificity and are not symptomatic of any physical disease or other psychiatric disorder. They can usually be elicited by taking a carefu history from the patient and also close family members and, if doubt remains, by careful observation of the patient's behaviour.

In the first two cases reported there is no mention of these cardinal features and presumably they were not looked for. In the third case it is said that there were "no food phobias and she saw herself as thin," and in the fourth case it is said that she "did not have a distorted body image." Thus there is no reason to suppose that any of these patients had symptoms of anorexia nervosa. Three of them are said to have presented with a history of "anorexia": despite the name anorexia nervosa is not characterised by poor appetite, although this may develop in advanced stages, and about half of all patients exhibit binges of overeating.

As doctors we tend to fear that the occasional physical disorder may be missed, even when there is no evidence for it on the basis of history and examination, and overinvestigation facilitates the process of somatisation of mental disorders, which is only now beginning to be recognised as a widespread problem in medical practice. The search for primary organic diseases in patients with anorexia nervosa delays recognition of the disorder and helps to endorse the views of patient and family that the main problem is physical. By contrast we do not appear to show equal concern about the possibility that mental illnesses may be missed when patients present with somatic complaints.

University Department of Psychiatry,

SIDNEY BENJAMIN Manchester Royal Infirmary, Manchester M13 9WL

\section{The need to make rugby safer}

SIR,-While spinal injuries from rugby are a cause for concern (16 January, p 149; 13 February, p 498; and 5 March, p 719), particularly when there are avoidable factors, these injuries should be viewed in perspective. Other sporting pursuits can be much more hazardous.

In our spinal injuries unit there have been only two admissions in the past 10 years because of injuries from rugby. There have, however, been 12 admissions after recreational diving accidents, five of which occurred while the victim was abroad on holiday. Most of these injuries were at unsupervised pools, beaches, or open water. Nine of these patients suffered complete spinal injuries, and three incomplete injuries, but all were at a high (C4-6) level. Our experience in this respect is similar to that of Young and Northup, ${ }^{1}$ who also indicate that diving injuries are much more common than rugby injuries.

A public education campaign is urgently required to emphasise the dangers of diving in unknown territory, particularly abroad and on holiday, when alcohol may play an important part, to try to reduce the incidence of these tragic injuries.

Neil W Valentine

West of Scotland Spinal Injuries Unit,

MARK A DELARGY

West of Scotland Spinal
Philipshill Hospital,

Glasgow G76 9HP

1 Young JS, Northup NE. Statistical information pertaining to some of the most commonly asked questions about spinal cord injury. Science Digest 1979;1:17.

\section{Diabetes and pancreatic cancer}

SIR,-Professor R C N Williams (13 February, $p 445$ ) suggests that the recent onset of diabetes or a sudden increase in the requirement for insulin in a diabetic should raise the suspicion of cancer of the pancreas and may offer a clue to early diagnosis That last claim, however, is unfortunately not supported by our experience.

A retrospective examination of the files of all patients with proved pancreatic carcinom diagnosed at our hospital in 1973-83 showed diabetes (two or more sequential fasting venou blood glucose concentrations of $\geqslant 7.2 \mathrm{mmol} / \mathrm{l}$ ) in 45 out of 74 patients compared with only eight out of 70 matched patients with carcinoma of the colon $(p<0.005)$. In 10 patients diabetes had been diagnosed more than two years before the pancreatic tumour (range 64-120 months, mean
64 months), in 11 less than a year before (range 0.5-12 months, mean $3 \cdot 3$ months, median 3 months), in 15 concomitantly, and in nine after the tumour was found (mean $5 \cdot 1$ months). All patients had locally unresectable or metastatic disease at the time of diagnosis. Most of the patients with recent, possibly tumour related, diabetes had only mild to moderate hyperglycaemia (less than $11 \cdot 1 \mathrm{mmol} / \mathrm{l}$ in 23 of 35 patients) and many were treated by diet alone. No relation was found between the development of diabetes and either the site of the tumour in the pancreas or its estimated size.

Thus the well known association between pancreatic cancer and the development of diabetes ${ }^{1}$ is even more striking in an Israeli population, but the belief that awareness of this association can not only serve as a diagnostic clue in patients with unexplained pain, weight loss, or jaundice but also aid an "early" diagnosis of potentially resectable disease seems overoptimistic, at least for the population we examined. The appearance of diabetes associated with pancreatic cancer usually heralds the more common and ominous signs by too short a time (median three months) to have any real impact on the patient's prognosis.

\section{A SCHATTNER}

Department of Medicine,

Kaplan Hospital and Haddassah Medical School,

Kaplan Hospital

Rehovo 7 Israel
I

1 Schwartz SS, Ziedler A, Moossa AR, Kuku SF, Rubenstein AH A prospective study of glucose tolerance, insulin, C-peptide and glucagon responses in patients with pancreatic carcinoma. Am F Dig Dis 1978;23:1107-10.

\section{Early growth delay in diabetic pregnancy}

SIR,-Dr Minna Bloch Petersen and others (27 February, p 598) suggest that children with a history of a first trimester crown-rump length smaller than expected from the menstrual history in diabetic pregnancy have an increased risk of developmental delay, although their results were significant only for language and speech $(p=0.009)$. It has previously been suggested that these fetuses may have experienced growth delay in very early gestation and have an increased risk of congenital malformation.

The existence of retarded fetal growth in very early gestation is difficult to prove unless the time of ovulation is known. Other studie ${ }^{2}{ }^{3}$ have shown that "early fetal growth delay" occurring in four of 11 diabetic pregnancies was explained by delayed ovulation (as judged from salivary progesterone levels) and that ultrasound measurement (of biparietal diameter between 12 and 18 weeks' gestation) is significantly better than an optimal menstrual history in predicting the date of delivery. ${ }^{23}$ We have found that in 56 insulin dependent diabetic mothers with certain menstrual data, regular 28-30 day cycles, and no recent contraception 11 fetuses had a crown-rump length (measured in the first trimester) which was six or more days less than expected from the menstrual data. Among these 11 fetuses the discrepancy between ultrasound and menstrual ages was explained in two by anencephaly; the other nine were normal and healthy at delivery with birth weights $>2500 \mathrm{~g}$. Of the 4 l fetuses whose ultrasonic and menstrual ages agreed, two had major cardiac malformations, three aborted spontaneously, and the remainder were normal and healthy at delivery with birth weights $>2500 \mathrm{~g}$. Our nine fetuses with "early growth delay" continued to grow at the rate expected had their ultrasonic ages been correct, as did those in the study of Dr Bloch Petersen and others, suggesting that, despite the rigorous inclusion criteria, ovulation in these pregnancies 